\title{
Clinical implementations of 4D pencil beam scanned particle therapy: Report on the 4D treatment planning workshop 2016 and 2017
}

\author{
Petra Trnkováa ${ }^{\mathrm{a}, \mathrm{b}}$, Barbara Knäusl ${ }^{\mathrm{c}}$, Oxana Actis ${ }^{\mathrm{d}}$, Christoph Bert ${ }^{\mathrm{e}}$, Aleksandra K. Biegun ${ }^{\mathrm{f}}$, \\ Till T. Boehlen ${ }^{\mathrm{d}}$, Hugo Furtado ${ }^{\mathrm{c}}$, Jamie McClelland ${ }^{\mathrm{g}}$, Shinichiro Mori ${ }^{\mathrm{h}}$, Ilaria Rinaldi ${ }^{\mathrm{i}, \mathrm{j}}$, \\ Antoni Rucinski ${ }^{\mathrm{k}}$, Antje C. Knopf ${ }^{1, *}$

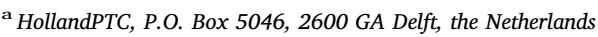 \\ b Erasmus MC, P.O. Box 5201, 3008 AE Rotterdam, the Netherlands \\ ${ }^{\mathrm{c}}$ Department of Radiation Oncology, Division of Medical Radiation Physics, Christian Doppler Laboratory for Medical Radiation Research for Radiation Oncology, Medical \\ University of Vienna/AKH Vienna, Austria \\ d Paul Scherrer Institute (PSI), 5232 Villigen, Switzerland \\ e Department of Radiation Oncology, Universitätsklinikum Erlangen, Friedrich-Alexander-Universität Erlangen-Nürnberg, Germany \\ ${ }^{\mathrm{f}}$ KVI-Center for Advanced Radiation Technology, University of Groningen, Groningen, the Netherlands \\ ${ }^{g}$ Centre for Medical Image Computing, Dept. Medical Physics and Biomedical, University College London, London, UK

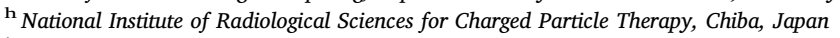 \\ ${ }^{\mathrm{i}}$ Lyon 1 University and CNRS/IN2P3, UMR 5822, 69622 Villeurbanne, France \\ ${ }^{\mathbf{j}}$ MAASTRO Clinic, P.O. Box 3035, 6202 NA Maastricht, the Netherlands \\ ${ }^{\mathbf{k}}$ Institute of Nuclear Physics PAN, Krakow, Poland \\ ${ }^{1}$ University of Groningen, University Medical Center Groningen, Department of Radiation Oncology, Groningen, the Netherlands
}

\section{A R T I C L E I N F O}

\section{Keywords:}

Proton therapy

Moving targets

4D imaging

4D dosimetry

\begin{abstract}
A B S T R A C T
In 2016 and 2017, the 8th and 9th 4D treatment planning workshop took place in Groningen (the Netherlands) and Vienna (Austria), respectively. This annual workshop brings together international experts to discuss research, advances in clinical implementation as well as problems and challenges in 4D treatment planning, mainly in spot scanned proton therapy. In the last two years several aspects like treatment planning, beam delivery, Monte Carlo simulations, motion modeling and monitoring, QA phantoms as well as 4D imaging were thoroughly discussed.

This report provides an overview of discussed topics, recent findings and literature review from the last two years. Its main focus is to highlight translation of 4D research into clinical practice and to discuss remaining challenges and pitfalls that still need to be addressed and to be overcome.
\end{abstract}

\section{Introduction}

Since 2009 the 4D treatment planning workshop is a well-recognized platform for detailed discussion on research and implementation of treatment planning approaches for moving targets in particle therapy $[8,55,56,58]$. In the past years, the scope and focus of the workshop has changed significantly. It is apparent that 4D treatment planning and delivery of pencil beam scanned particle therapy is slowly moving from "science fiction" to clinical reality. Therefore, more and more discussions during the meeting address the challenges of clinical implementation of current technologies. The research and development also evolved from investigating the elementary proof of principle to expanding and advancing the current state-of-art technology.

To be able to describe the motion and to deliver conformal treatments with small margins to moving targets without partial tumour misses, motion monitoring is crucial. As discussed during prior workshop reports $[8,56,58]$, all 4D approaches, especially motion monitoring and mitigation techniques have been generally pioneered in photon therapy while their application to protons and ions was usually following in their footsteps with some delay, but catching up quicker in recent years. The faster advancement of clinical 4D photon technology is owed in part to much larger patient numbers and hence a larger community and commercial interest but also due to its easier delivery method as well as the different physical interaction mechanism of photons with human tissue. Due to the higher dose conformity of

\footnotetext{
* Corresponding author.

E-mail address: a.c.knopf@umcg.nl (A.C. Knopf).
} 
proton and ion beam depth-dose profiles, a superior accuracy and precision of motion monitoring and mitigation techniques is needed in order to apply these beams safely. Actually, both delivery techniques would benefit from an improved motion monitoring accuracy but due to the finite range of charged particles, potentially more accurate adaptations in 3 dimensions could be performed compared to adaptations in 2 dimensions for photons. Interplay effects between beam and target motion complicate additionally the delivery for scanned proton and ion beams and may lead to inhomogeneous dose distributions $[9,52]$.

The 8th annual workshop took place in Groningen, the Netherlands. After some years of exclusion, representatives of commercial vendors were allowed again to join the audience. Each year, the decision to include or exclude representatives of commercial vendors is taken by the local organizers, balancing the opportunity for unbiased and completely open scientific discussion against the importance to feed back the need for certain developments to industry. The talks addressed clinical experience, treatment planning, experiments and delivery, motion modeling and monitoring. In this report, a talk of Tony Lomax (Paul Scherrer Institute, Villigen, Switzerland) on treatment planning strategies is highlighted. His key message was that even though technologies on the treatment of moving targets are advancing, the basic principles, like the choice of the beam angle should not be forgotten, as they might be the essential parameters for safe 4D treatments. The poster price 2016 was awarded to Jennifer Dhont (Universitair Ziekenhuis Brussel, Brussels, Belgium) for "Variability of breathing-induced tumor motion: 4DCT - a source of misguiding information?".

Vienna, Austria, hosted the 9th annual workshop. The topics were focused on the experience in the clinical implementation. The talk of Jan Hrbacek (Paul Scherrer Institute, Villigen, Switzerland) on perspective of medical physicist when starting clinical treatments of the moving targets will be highlighted in this report. A new topic of artificial intelligence driven adaptive radiation therapy was introduced by Nikos Paragios (Ecole Central Paris, Paris, France). The poster price for 2017 was awarded to Christopher Kurz (Ludwig-MaximiliansUniveristät München, Munich, Germany) for "An ex-vivo porcine lung phantom study to assess the feasibility of $4 D C B C T$-based proton dose calculation" being representative for the strongly discussed problem of the accuracy of 4D imaging during this workshop.

The aim of this report is to provide a comprehensive overview of the current status of treating moving targets in particle therapy. The main focus is on the discussion of problems that arose during this phase of first clinical implementations. Furthermore the aim is to broaden the knowledge on ongoing research in the field.

\section{Current status of 4D particle therapy}

Despite all openly discussed challenges with actively scanned proton beams in combination with motion, an increasing number of centers is starting to use actively scanned proton beams for sites in body regions prone to movements, such as lung, liver and thorax [58]. This trend is primarily fueled by the benefit of the conformal dose distribution of proton therapy, potentially providing a promising possibility to precisely treat the target while sparing healthy tissue, in combination with the fact that new proton centers tend to install dedicated active beam scanning rooms only. However, the clinical implementation is hampered by patients with large or irregular motion amplitudes, the precision of currently available motion characterization methods, unfavorable beam delivery characteristics (as for example pulsed beams, scanning speed or spot sizes), 4D treatment planning capabilities, the availability of motion mitigation techniques and shortcomings in $4 \mathrm{D}$ imaging.

4D imaging, motion monitoring and motion modeling are essential for treatments of moving targets. Currently, a major focus in the radiotherapy community (with protons and carbon ions) is the improvement of in-room imaging capabilities. A few centers are meanwhile equipped with in-room CT, allowing for imaging in the treatment position or even with online CBCT, allowing for imaging at the iso-center. In-room imaging will enable motion acquisition just prior treatment delivery, improving motion modeling. In recent years, more and more studies on magnet resonance (MR)-guided proton therapy have been published $[94,118,80,43,85,32,86]$, envisioning new ways enabling visual tracking of the tumor by imaging and tracking the tumor with the treatment beam. Chapter 3, 4 and 5 of this report will give more insights on the current challenges faced in $4 \mathrm{D}$ imaging, motion monitoring and motion modeling.

4D treatment planning in many facets (e.g. constructing ITVs, optimizing with taking into account different 4DCT phases, probabilistic planning) has been practiced in photon therapy for years $[87,96,106,113,68,67,15,62]$, but only recently it has become commercially available for proton therapy. Only a few studies about 4D optimization in particle therapy have been published $[39,38,13,24,81,25]$. Chapter 6 of this report is dedicated to the current experience with $4 \mathrm{D}$ treatment planning for moving targets.

Beam delivery characteristics differ from particle center to particle center. Development proceeds further towards faster scanning speeds, higher dose rates and smaller spot sizes. In chapter 7 , advantages and disadvantages of different beam delivery characteristics will be discussed together with new developments.

Assuring an accurate and predictable dose delivery is most challenging for moving targets raising the demand for highly sophisticated anthropomorphic 4D phantoms in order to perform experimental validation of upcoming techniques and end-to-end testing before clinical implementation. In recent years, several research groups developed prototypes of those phantoms that can be used in both, ion beam therapy and photon therapy. In chapter 8 of this report, an overview of recently developed 4D phantoms is given and demands on future evolutions are discussed.

New technologies, like machine learning or artificial intelligence open new possibilities in the field of motion modeling and deformable image registration. These new perspectives are discussed in more details in chapter 9 of this workshop report.

In addition to this report, the status quo on treating mobile tumors with scanned proton beams is also well reflected in the recently developed guidelines of the Particle Therapy Co-Operative Group (PTCOG) Thoracic/Lymphoma Subcommittee, which is based on available physics and clinical findings, for the use of intensity modulated proton plans (IMPT) for thoracic tumors [14]. Furthermore, a review article by Diwanji et al. highlights recent advances in radiotherapy techniques and delivery for non-small cell lung cancer [19].

\section{4D imaging}

Accurate $4 \mathrm{D}$ imaging is the basis for $4 \mathrm{D}$ dose calculation as well as for building motion prediction models. 4DCT imaging is essential for providing the density information for dose calculation and will remain the mainstay of clinical 4D planning in the coming years. However, its benefits are limited, among others, by a low temporal resolution, the inability to capture motion variations and its restriction to off-line acquisition (not in treatment iso-center position). 4DMRI and 4DCBCT have the potential to overcome these limitations in the future.

Standard 4DCTs have a low temporal resolution, which is usually in the order of $0.8-0.4 \mathrm{~s}$ per respiratory gate, and long acquisition times due to the limited scan range within a single rotation. Therefore, they are prone to motion artefacts resulting in deformation of critical structures. A 4DCT most of the time requires optical, load cells or spirometric motion surrogates in order to establish the anatomical correlation. Research has been published on Iterative sorting for fourdimensional CT images based on internal anatomy motion [123] and a commercial implementation for a "smart deviceless 4D" reconstruction is nowadays available from GE Healthcare [35]. Pre-treatment 4DCT allows to visualize target displacement caused by breathing and to 
evaluate if the tumor moves independently of the thorax [116]. When performing 4DCT based 4D dose calculation, the dose distribution on a single (reference) CT is accumulated summing up the dose distributions calculated on each individual image sets for different breathing phases $[9,131]$. However, irregularities in breathing, which frequently occur in real patients and which are responsible for geometrical uncertainties and misrepresentation of tumor trajectory, are not properly represented by 4DCTs [60].

To build accurate motion models, the imaging data should give a good representation of the true motion with potential variations. 4DCT data has often been used to build motion models, however, it only gives average information about the breathing motion. In contrast to 4DCT some 4DMR methods can produce images showing variations in the breathing motion [115]. To improve the time resolved dose calculation, one can deform the calculation dose grid using deformable vector fields from 4DMRIs [125,11] including motion variations. While 4DMRI images are better than 4DCT for building motion models and performing time resolve 4D treatment planning, they require long acquisition times, and are created by sorting the data acquired during different breath cycles retrospectively. Therefore, they may still not give a perfect representation of the true breathing motion. Ideally, the motion models would be built from 'true' 4D imaging data, which can acquire a full 3D image fast enough to 'freeze' the breathing motion. Currently true 4D imaging methods either suffer from poor image quality and/or a restricted field-of-view. An alternative approach is to fit the models directly to the 'partial' or 'raw' imaging data, such as individual slices or projections. This way, sorting or binning to form images can be omitted prior to fitting the model [72].

In order to include the inter-fraction and intra-fraction variations in amplitude and phase, the internal motion pattern created on the basis of 4DCT should be updated before each treatment session with reference to the data from in-room radiography and surface imaging $[3,28,78]$. With more proton centers equipped with CBCT, also 4DCBCT will become a choice for pre-treatment probing of breathing pattern and anatomy changes. A poster about "An ex-vivo porcine lung phantom study to assess the feasibility of 4D CBCT-based proton dose calculation" by Christopher Kurz et al. from Munich won the poster price of the 4D workshop 2017. The group from Munich investigated the feasibility of using CBCT data for accurate 4D pre-treatment imaging and proton dose calculation. With this research they pave the way for $4 \mathrm{D}$ adaptive proton therapy based on $4 \mathrm{DCBCT}$.

While 4DCTs do contain many pitfalls and errors, they remain the mainstay also of clinical 4D planning and this will also not change in the foreseeable future. They contain valuable information, especially if combined with some form of motion training to achieve regular breathing [92]. Artifacts can potentially be significantly reduced with modern multi detector CTs (MDCTs). A recent study by Behzadi et al. reports that high image quality can be acquired using respiratory gating techniques for 4D-MDCT of the thorax [4].

\section{Motion monitoring}

Motion monitoring is essential during 4D imaging as well as during treatment delivery and ideally identical setup should be used for both. In this section however, we refer to motion monitoring as tracking of the motion states directly before and/or during fraction delivery. The talk of Guido Baroni during the 4D workshop 2017 about "Which techniques are really used for motion monitoring" highlighted the lack of research in the particle therapy world in this field. During the talk, a comprehensive overview about available motion monitoring techniques and their drawbacks was given. It was pointed out that for treatment planning, 4DCT might be considered a standard technique but the general consensus is that there is the need of improving its quality and time resolution. Furthermore it was stated that for proton therapy tumour tracking is probably not sufficient due to range uncertainties. Motion modelling may turn out as a great tool supporting adaptive strategies but deformable image registration (DIR) algorithms have to be treated with caution in this context.

Techniques for motion monitoring generally capture reduced 4D data sets and need to correlate the acquired motion signals either to the 3D planning CT (gating) or to a state of the 4D planning CT (tracking). It is convenient to discriminate between monitoring techniques which image directly internal motion and surrogate motion. In particular, the monitoring of respiratory motion often relies on a surrogate motion, which is assumed to be correlated to the actual tumour motion. As highlighted by a poster from Dhont et al. with the title "Variability of breathing-induced tumor motion: 4DCT - a source of misguiding information?", respiratory tracking data from clinics show, however, that direct monitoring and real-time adaptation is crucial for some cases. Irregularities, such as frequency and baseline shift, may occur randomly and cannot be fully captured by surrogates combined with predictive models. Regular updates to realign predictive models to eventually occurring irregularities (e.g. confirmation of surrogacy with fluoroscopy) can be a viable intermediate solution.

The previous workshop report covered recent advances and developments of motion monitoring using ultrasound and magnetic resonance imaging [58]. In the workshops of 2016 and 2017 a focus was set on clinical usage of monitoring techniques in both photon and ion beam therapy centres. A vast variety of motion monitoring systems are presently used clinically. In Table 1, a summary of established monitoring systems is given together with some of their characteristics. Nearly all of the systems provide integration with photon linacs. Some of them also provide a dedicated interface to commercial proton therapy systems. The applicability and performance of tracking methods is strongly site specific. As of today, no clinical guidelines and indication-specific standardization for motion monitoring techniques for scanned ion beams exist. It is important to recognize that the clinical feasibility and overall performance of a specific monitoring method is often heavily dependent on the integrated data processing algorithms, including for instance automatized contour/marker recognition and surrogacy motion models. Due to their reliability and ease-of-use, wellrecognizable point-like and 1D signals currently prevail clinically. This picture might be somewhat altered in near future with the advancement of segmentation, image processing, motion modelling and the advent of additional advanced techniques, such as machine learning. Invasive techniques using implanted high-density markers generally lead to sizeable beam range uncertainties, as well as additional target heterogeneity and CT artefacts. This makes their usage for ion beams often suboptimal.

Several works on emerging imaging technologies for proton and ion beam range prediction and monitoring were presented at the 2016 and 2017 4D workshop. It was shown that ion radiography [112] could be acquired with the patient in treatment position just before every fraction, to perform an on-the-fly pre-treatment patient-specific plan reoptimisation. A poster by Rinaldi at al. showed that also during a single treatment fraction ion radiographs could be acquired to track internal motion, by interleaving the treatment plan with ion beams of a higher energy than the one used for treatment to irradiate the region of interest to be tracked. Other contributions showed that ion beam therapy range monitoring can be realized by back-tracking and reconstructing the emission profile of charged secondaries [91]. The possibility of online ion range prediction in tissues and the derivation of internal target motion information from charged secondary emission profiles was presented in a poster by Rucinski et al.

Starting from a list of motion-related problematics, a practical attempt has been made to classify adequacy and performance of the versatile and heterogeneous landscape of motion monitoring techniques from a clinical bottom-up perspective. Objectives of motion monitoring considered are: (1) monitoring of tumour motion, (2) monitoring of surrounding tissue motion, (3) intra-fractional surface variation (such as a twitched chest wall), (4) inter- and intra-fractional tumour/organ deformation (such as tumour shrinkage, bladder filling), (5) density and 


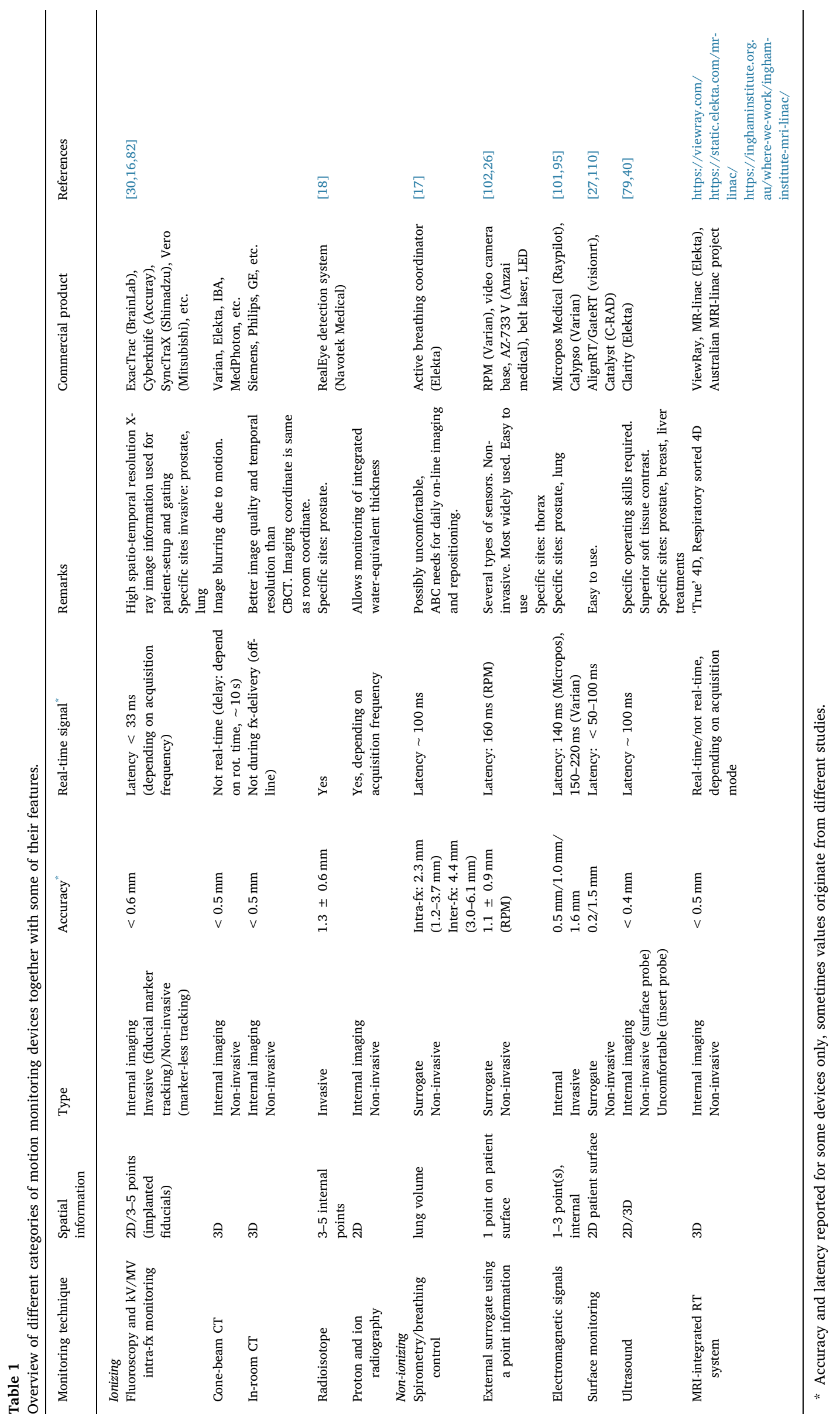


Table 2

Capability and performance of motion monitoring techniques to address specific motion-related issues (subjective view of the authors).

\begin{tabular}{|c|c|c|c|c|c|c|c|c|}
\hline Monitoring technique & $\begin{array}{l}\text { (1) Tumour } \\
\text { motion }\end{array}$ & $\begin{array}{l}\text { (2) Surrounding } \\
\text { tissues motion }\end{array}$ & $\begin{array}{l}\text { (3) Intra-fx } \\
\text { surface } \\
\text { variation }\end{array}$ & $\begin{array}{l}\text { (4) Tumour/organ } \\
\text { deformation }\end{array}$ & $\begin{array}{l}\text { (5) Density } \\
\text { and range } \\
\text { changes }\end{array}$ & $\begin{array}{l}\text { (6) Irregular } \\
\text { breath/base line } \\
\text { shifts }\end{array}$ & $\begin{array}{l}\text { (7) Inter-fx } \\
\text { motion/anatomy } \\
\text { changes }\end{array}$ & $\begin{array}{l}\text { (8) External/ } \\
\text { internal } \\
\text { correlation }\end{array}$ \\
\hline $\begin{array}{l}\text { Fluoroscopy and } \mathrm{kV} / \mathrm{MV} \\
\text { intra-fx monitoring }\end{array}$ & $++(1,7)$ & $+(1,8)$ & - & o $(1,8)$ & - & $++(1)$ & $+(1,7,8)$ & $++(1)$ \\
\hline Cone-beam CT & o (2) & o (2) & - & $++(2)$ & $++(2)$ & - & ++ & - \\
\hline In-room CT & $+(3)$ & $+(3)$ & - & $++(3)$ & $++(3)$ & - & ++ & - \\
\hline Radioisotope & ++ & - & - & - & - & - & ++ & - \\
\hline $\begin{array}{l}\text { Proton and ion } \\
\text { radiography }\end{array}$ & - & - & - & - & ++ & - & - & - \\
\hline $\begin{array}{l}\text { Spirometry/breathing } \\
\text { control }\end{array}$ & $+(4)$ & $+(4)$ & - & - & - & o & - & - \\
\hline $\begin{array}{l}\text { External surrogate using a } \\
\text { point information }\end{array}$ & $+(4)$ & $+(4)$ & - & - & - & o & - & - \\
\hline Electromagnetic signals & ++ & - & - & + & - & ++ & ++ & - \\
\hline Surface monitoring & o (4) & o (4) & ++ & & - & o & - & - \\
\hline Ultrasound & $++(5)$ & $++(5)$ & - & $++(5)$ & o (5) & + & $++(5)$ & + \\
\hline MRI-integrated RT system & $++(6)$ & $++(6)$ & $++(6)$ & $++(6)$ & o (6) & $+(6)$ & $++(6)$ & $++(6)$ \\
\hline
\end{tabular}

++ : good, + : possible, o: principally capable, -: not capable/not applicable.

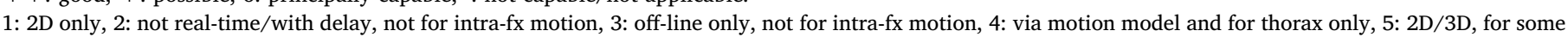

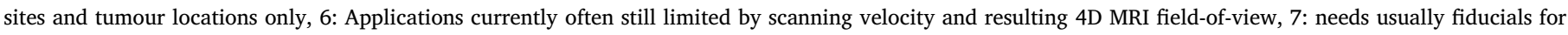
tumour visualization, 8: often tissues/tumour are not directly visible.

related range changes for ion beams, (6) irregular breathing patterns and baseline shifts, (7) inter-fractional motion and anatomy changes, and (8) correlation of external surrogate motion with internal anatomy. A summary of the capabilities of the different techniques is shown in Table 2. The table illustrates that the choice of the adequate monitoring system is highly purpose- and site-specific. Often a combination of several methods is needed to achieve motion mitigation in the clinics.

Motion takes place on a range of time scale during a course of radiotherapy - inter-fractionally from day to day (for instance due to weight loss or changes in organ functionality), and within minutes and seconds during a treatment fraction (for instance due to bladder filling and respiration).

The monitoring of respiratory motion often relies on a surrogate, the motion of which is assumed to allow the derivation of the actual tumour motion. Monitoring of respiration through a surrogate can involve a diversity of techniques (i.e., optical recording, spirometry, thermocouple, piezoelectric ceramics). All these surrogates give a respiratory cyclic signal reflecting the period (and phase) of breathing as well as of some measure of the magnitude of respiration. Common and inherent for all the surrogates is the fact that they do not give the actual tumour/ target position which must be derived.

\section{Motion modeling}

Respiratory motion models relate the internal motion of interest to one or more easily measured surrogate signals. The surrogate signals can be 'external signals' such as markers on the skin surface or spirometry, or 'internal' signals such as the motion of the diaphragm [71]. These models have much potential used in both, particle and photon radiotherapy, as well as in many other medical applications. They can be used to compensate for motion during image acquisition [97], to account for motion when planning treatment [7] and to guide the treatment delivery [108]. So far such models have seen a lot of interest in the research literature, but relatively little clinical use. The main exceptions being the Cyberknife (Accuray Inc. Sunnyvale, CA, USA) and Vero (Mitsubishi Heavy Industries, Ltd., Tokyo, Japan and Brainlab AG, Feldkirchen, Germany) systems, which generally require implanted markers to be used and only model the motion of the tumor (as represented by the markers). There have also been some limited clinical use of motion models to compensate for motion during the acquisition of CBCT data [97] and '5DCT' data [114]. There are still challenges that must be overcome to see a more widespread clinical use of motion models.

One remaining challenge is the validation of the motion models. The problem is that for real subjects the ground truth motion is not known. Hardware and software phantoms can be used, where the ground truth motion is known, but there will always be questions over how realistic the phantoms are, and how applicable the results are to model the real human anatomy. The models can also be validated using imaging data from real subjects, ideally acquired after the imaging data used to build the model. It may be possible to identify particular structures or landmarks in the data which can be used to validate the models; however this becomes difficult when using partial or raw image data such as slices or projections.

Another challenge that needs addressing is the trade-off between how complex the models need to be (and hence how much motion variability they can potentially account for), how much data is required to fit them, and how often they need to be updated or rebuilt to maintain an accurate representation of the motion. Furthermore, the question of which surrogate signals are best to use, and for which applications (e.g. the surrogates that can be acquired in a proton gantry and different to those which can be acquired in an MR-Linac) should be answered. As many of the models use image registration to determine and represent the motion there are also questions about which registration algorithm(s) should be used. And finally the best ways of using the motion models to correct different types of images and to plan and guide different treatment deliveries need to be established.

Although there is lot of remaining work to be done for motion models to reach their full potential, it is worth bearing in mind that it may not be necessary to find perfect solutions to all these issues before motion models become more clinically adopted. It is generally agreed that the motion seen in most 4DCT datasets is not the true motion that the patient undergoes during treatment delivery, but it is the best approximation that is available. If it can be demonstrated that motion models give a better approximation of the true motion, and if their limitations and uncertainties are understood, then they could have a promising future in radiotherapy and other applications.

\section{4D treatment planning and motion management}

Effects of the movement of the target and the surrounding tissue on the dose delivery, like dose blurring, interplay effects during spot scanning as well as range changes must be taken into account during the $4 \mathrm{D}$ treatment planning process. The treatment planning approach is 
dependent on the availability of the methods described in previous paragraphs. The simplest possible approach if none of these methods is available is to pay attention to a patient selection. E.g. [122] addressed that targeting the right patient for proton therapy can help with motion problems. Moreover, the motion effects can be minimized if the beam selection is as close as possible to be parallel to the motion direction. Bernatowicz et al. [6] performed 4D simulation study in liver showing that target homogeneity can be improved by optimizing gantry angle selection.

Another simple approach is limiting the motion by either abdominal compression systems or breath hold techniques. Comparing treatment plans for free breathing and compression using 4DCT showed significant control of lower lobe lung and liver tumors when using abdominal compression [44]. A similar study using cine-MRI came to the conclusion that abdominal compression reduces liver motion in most patients, although the magnitude of the reduction was smaller than previously reported [21]. The main disadvantage of abdominal compression is a limitation of possible gantry angles, as passing through the device should be avoided. On the other side, breath hold techniques enable full range of gantry angles to be used and provide increase patient comfort. However, it was reported that remaining tumor motion can be observed during the breath hold, depending on the tumor location and breathing phase $[90,57]$. It was shown that using exhalation breath holds rather than inhalation breath holds and delaying irradiation until after the first $10 \mathrm{~s}$ of breath-holding might be advantageous for irradiation of abdominal tumors [61]. One step further would be active motion management by mid-ventilation which can lead to additional improvements in dose distribution [22].

An alternative approach is the use of an internal target volume (ITV) based on 4D imaging. However, the ITV approach is able to deal with dose blurring. But neither interplay effects nor range changes can be fully solved with this approach. Robust optimization on the target volume instead of an ITV can take into account the range changes but interplay effects must still be solved by more advanced techniques. Liu et al. [65] have conducted an exploratory methodology study. This study showed that compared to 3D robust optimization, 4D robust optimization produced significantly more robust and interplay-effectresistant plans with comparable target coverage and dose distributions for normal tissues. However, it is challenging to predict the impact of the interplay effect on the dose distribution during the 4D treatment planning process due to varying beam intensities and organ motion period irregularities. Therefore, retrospective dose calculation based on online acquired motion signals (e.g. from an ANZAI belt system), machine $\log$ files and weekly repeated 4DCTs/daily repeated 4DCBCTs should be performed, possible enabling 4D adapted proton treatments in the future as highlighted in the talk by Richter at al. at the 4D workshop 2017. An approach called dose restoration that works along the same lines approaching online IMPT adaptation was recently published by Bernatowicz et al. [5]. To reduce the effect of interplay, rescanning can be applied. In addition, gating or tracking during the delivery are suggested. While gating is already used clinically in some centers, tracking remains a research topic, at least in the particle therapy world.

An important factor for the quality of 4D treatment plans is determined by the dose calculation engine itself. Recent publications claim that pencil beam algorithms are unsuitable for proton dose calculations in lung [111]. Monte Carlo (MC) dose calculation engines are becoming widely available and less computational demanding and therefore can be much easier implemented clinically [88]. MC simulations calculate the transport of ion beams, especially proton and carbon, but also a beam of electron and photon in heterogeneous patient tissues, considering interactions of the primary particles with electrons and nuclei of various materials (tissues) in a wide range of energies and cross-sections $[2,20]$. This results in an increased accuracy with respect to analytical dose calculation methods. RaySearch (https://www. raysearchlabs.com/raystation/) released the first commercial TPS making a MC dose calculation algorithm available for particle therapy which was experimentally proved to work well for clinical challenging scenarios [99] while Varian followed with the implementation of AcurosPT in Eclipse [63].

In the future, also novel applications, such as time resolved dose calculation that are needed to accurately model dose distribution in moving targets, should employ 4D MC dose engines [48,49,53,98]. Very fast MC-based dose calculations have the potential to perform several dose optimizations and recalculations in a clinically acceptable short time. In most implementations these codes run on a CPU cluster and it takes few hours to calculate the dose distribution, which is too slow for a clinical use. To meet the clinical requirement, calculation time in minutes, the use of graphics processing unit (GPU) technology have been considered and investigated for fast MC-based TPS [50]. An example for a fast MC implementations is the gPMC code developed by Fracchiolla et al. [31]. With gPMC it takes only up to $22 \mathrm{~s}$ to simulate 10 million protons from the source to achieve $\sim 1 \%$ relative statistical uncertainties. During the 4D workshop in 2016, another fast MC dose engine, called MCsquare (many-core Monte Carlo), was presented [109]. It uses parallel computation architecture and allows to accurately simulating a therapeutic plan in less than one minute. MCsquare has been benchmarked with the GATE/Geant4 MC application (http:// www.opengatecollaboration.org/) and could be used as an efficient tool for dose (re)calculations on the day of the treatment in combination with OpenReggui (https://www.openreggui.org/). An independent development of the research version of a MC-based TPS, called FRED, is being developed and benchmarked by Schiavi et al. [105]. The tracing kernel in FRED can run on GPU hardware, achieving 10 million primary $\mathrm{s}^{-1}$ on a single card. This performance allows one to recalculate a proton treatment plan at $1 \%$ of the total particles in just a few minutes.

\section{4D beam delivery}

Treatment of moving targets requires improvement of the existing beam delivery systems and implementation of new delivery techniques. Motion mitigation methods such as rescanning, gating, breath-hold and tracking can be implemented only with highly dynamic, fast and precise (in terms of beam position) delivery systems. The main technical challenges addressed to beam delivery systems as well as possible solutions in order to overcome those challenges were discussed during these workshops.

Nearly all hadron therapy facilities use synchrotrons or cyclotrons to generate clinical beams. All presently operating carbon-ion facilities use synchrotrons which are capable of accelerating a variety of ions suitable for cancer treatment, while proton therapy facilities often choose cyclotrons due to their compact design and relatively low cost. Especially compact $(\sim 3.5 \mathrm{~m}$ diameter) are cyclotrons designed using superconducting magnets.

New developments in the field of accelerators for medical applications focus on improvement of the performance and reduction of the therapy system size. A disadvantage of synchrotrons is a pulsed beam extraction (spills). The acceleration time (which is typically used to switch the energy) can vary from 1 to $10 \mathrm{~s}$. A new synchrotron solution developed at HIMAC [84] is capable of changing energy multiple times within one spill $[47,76,42]$ resulting in continuous beam extraction and significant reduction of the total treatment time. In addition, Sato et al. [100] has shown that the intensity control of a synchrotron [34] can be performed within less than $1 \mathrm{~ms}$ time. Both parameters are important for implementation of new scanning techniques which are expected to be advantageous for moving target treatment [103,54]. Another synchrotron-based facility which operates a carbon-ion gantry, HIT, does not have a possibility to change the energy within one spill but implemented the dynamic intensity control and uses it in clinical standard operation [107].

Cyclotrons, instead, are designed to accelerate only one type of particles, but offer almost continuous beam extraction releasing protons 
every $\sim 30 \mathrm{~ns}$. This type of accelerators is compatible with continuous (line) scanning, implemented by Sumitomo Heavy Industries, Ltd. and at PSI [103]. In comparison to pencil beam scanning (PBS), line scanning allows avoiding dead time between spots resulting in a reduction of the total dose delivery time.

For PBS, the most studied method to mitigate the dose delivery uncertainties due to the organ motion is rescanning [124,104,93]. Rescanning by itself is triggering additional irradiation times and requires the deliverability of low spot weights. Bernatowicz et al. [6] and Zhang et al. [126] compared layered and volumetric rescanning for different scanning speeds and studied the effectiveness of rescanning in combination with gating. The combination of different motion mitigation techniques promises to be the most effective strategy for tumors with larger motion amplitudes.

Rescanning alone and in particular combined with gating extends the total irradiation time and therefore the focus of many research groups is on the improvement of beam delivery efficiency. The research groups from HIT and GSI developed a scan path optimization algorithm which together with a dynamic beam intensity control leads to a significant reduction of irradiation time in particular for re-scanned highdose irradiations [23]. In order to shorten the treatment time for gated irradiation with rescanning the research group at NIRS developed an extension to the existing treatment planning algorithm where the effect of the beam-intensity-dependent extra dose due to raster scanning is integrated into the optimization process [46]. The new studies focus on determination of the patient specific maximum intensity, which would allow for a fastest possible irradiation keeping the dose in conformity with tolerance criteria.

Up to now, there is no standard quality assurance (QA) procedure for centers employing PBS-PT and each center applies a specific QA program [1]. Patient-specific quality assurance (QA) for PBS-PT, especially IMPT, is challenging when treating moving targets. Currently, QA measurements are mainly made with a 2-dimensional ion chamber array detector for PBS treatment fields [130,129,64] and 3D detectors are being developed [37,66,127]. NIRS developed new dosimetry equipment to efficiently perform patient specific QA [33,77]. After intense commissioning and quality assurance tests, the treatment of moving target was started in March 2015. To further automate patientspecific QA new hardware and software solutions for a highly efficient clinical operation are needed. One possibility could be to replacing patient specific QA for pencil beam scanned proton therapy with Monte Carlo simulations [117]. To treat moving targets with a PBS technique, motion interplay effects and the effectiveness of motion mitigation strategies should be evaluated before patient treatment. The use of a 4D phantom is recommended for this purpose. Developed and available 4D phantom designs are discussed in Section 8 of this review. If an adaptive plan is deemed necessary during the course of treatment, the same patient-specific QA process should be repeated before treatment with the adapted plan.

\section{4D phantoms}

A cutting-edge 4D phantom must mimic an average human torso consisting of tissue equivalent components. An independent motion of the chest wall and ribs, the lungs and the tumor are the precondition for realistic experiments. Ideally, those motion can be based on real-patient breathing patterns or alternatively on programmable mathematical functions and need to be loaded in reproducible way. The external phantom motion must be easily detectable by surface scanning or other motion surrogates to test triggered beam delivery. The possibility to use different dosimeters like TLDs, films or ionization chambers should be guaranteed, ideally in 3 dimensions. Since the ideal 4D phantom is not only suitable for dosimetric measurements but also for testing image registration software, it should be possible to insert an individual tumor shape. Imaging computability with in-room fluoroscopy, 4D-CT and 4DPET and well as MR are ideal pre-conditions for a wide-spread applicability. In the context of recent developments and improvements in $4 \mathrm{D}$ imaging, as described in chapter 3 , dedicated phantoms are also developed, which include highly sophisticated independently moving, deformable components like lungs, liver and the cardiac unit [10].

Commercial solutions are behind with the development concerning complex movements and customization. The most advanced commercially available phantoms are the dynamic thorax phantom from CIRS (CIRS Dynamic Thorax Phantom, MR-compatible version available), the QUASAR phantom (QUASAR Cylindrical Respiratory Motion Phantom, MR-compatible version available) and the Dynamic Alderson Phantom (RS-1500 Breathing Phantom System). All of these phantoms are made from anthropomorphic components that can move independently. Individual adaptation of these commercial phantoms is getting more and more common, e.g using 3D printed inserts for dosimeters [12]

The most recent developments for 4D phantoms include the socalled LuCa phantom from the Paul Scherrer Institute [89]. In this phantom, the lungs were mimicked by inflatable and deformable opencell foam. Lungs could include veins and bronchioles for 4DMRI deformation-tracking purposes being able to be steered by arbitrary and varying pressure defined motion patterns.

Another 4D phantom was developed within the Framework 6 European project "Methods Advanced Equipment for Simulation and Treatment in Radiation Oncology" (MAESTRO) [41]. This phantom contains eight independently reproducible moving rib pairs surrounding two stationary lungs filled with water with a spine in the middle, all covered by skin. The tumor in this phantom can be equipped with TLDs, ionization chambers or film holders. A research group in Erlangen created a phantom with new synthetic tissue equivalent materials [120] especially suitable for the use in particle therapy. An independent tumor motion in all three directions as well as a thorax movement can be facilitated on the basis of arbitrary movement curves while inserts for films or ion chamber are available. Another sophisticated research phantom was developed at the Medical University of Vienna in cooperation with the Austrian Institute of Technology an Advanced Radiation DOSimetry phantom (ARDOS) in the frame of a Marie Curie project [59]. This phantom was built from three different tissue equivalent materials: high density balsa wood for lung, solid water for soft tissue and bone material. The tumor insert is suitable for films, TLDs or ionization chambers. The movement of the torso was simulated by the conical shape moving into cranio-caudal direction. Independent sinusoidal and realistic motion curves can be simulated with maximal amplitudes beyond the clinically reported breathing amplitudes. Due to the out-off axis position of the tumor insert and the rotational movement of $360^{\circ}$ a movement trajectory can be described by the tumor.

The future of the 4D phantoms is in new material compositions, including approaches to build phantom components from 3D printing materials on the basis of real lung patient CT scans [70]. Also creating phantoms which partly consist of (preserved) real tissue, e.g porchine lungs which can be externally inflated [69] are more and more popular. This is especially used for $4 \mathrm{D}$ imaging, segmentation and registration validation but have the disadvantage of having a short lifetime.

Despite all the sophisticated phantoms it needs to be kept in mind that for $4 \mathrm{D}$ dose calculation validation, especially when being in a development phase, a simple moving platform shows to be essential and a low-cost solution [81].

\section{New perspectives}

One of the most interesting and exciting developments of recent years is the application of machine learning (ML) approaches to image guided adaptive radiation therapy. At the 4D workshop in 2017 the talk by Nikos Paragios showed an overview of possibilities and opportunities in this field. He highlighted how radiotherapy could profit from machine learning, transfer (anatomy-consistent) learning, reinforcement learning, parallel optimization algorithms and parallel physics- 
based simulation algorithms. Main developments he foresees would include tools for automatic segmentation, tools for coupled real time simulation \& dose optimization, tools for automatic positioning towards optimal constraint satisfaction, tools for cumulative dose estimation enabling automatic re-planning and eventually the realization of radiation therapy on the fly.

On a conceptual level, machine learning is an approach to program and optimize a mathematical model enabling a computer to replicate human perception. In general, the main idea consists of iteratively modifying the model approach based on annotated training data i.e. on presenting the model with a large amount of data and, the ground truth about that data, and update the model accordingly so that new observations can be correctly classified [75].

The applications are countless ranging from annotation [36], segmentation [45], registration [74], synthetic CT generation [119], plan quality assessment [83] among many others. Some of the most promising used cases are deformable registration and dose accumulation [29] and individualized patient treatment based on heterogeneous data [51].

While it is tempting to use ML for just about everything, care should be taken to understand what it can or cannot do and to use it when appropriate. One of the key points always lies in finding out exactly which part or parts of conventional algorithms can be approached with ML techniques. As an example, the framework to tackle (deformable) registration problems typically consists of a model to deform a moving image into the fixed image, a metric to measure how similar the deformed and fixed image are and an optimization part which loops until the metric shows good agreement. For challenging multi-modal datasets, it is not clear which metric is the best to compare images. An interesting approach is to use a linear combination of conventional metrics. The best combination depends on the context i.e. on the type and region within the images where the similarity is being measured. Because finding the optimal linear combination is not obvious, deep learning techniques [73] can be used, to learn the best combination of these context-specific similarity measures. The weights of the linear combination can be learned using a support vector machines framework [121].

This example and many others are made possible by the recent and continuous improvements in data collection possibilities and processing power. In particular, the current opportunity of systematically collecting massive amount of data is invaluable. Quality data is a necessity to generate meaningful models. However, it is still very challenging to generate big amounts of quality data due to the lack of standards, relative low incident numbers per institute and rapidly evolving technology.

The generation of good models is only possible due to the massive parallel computation power that is available today and that will continue to increase. The further developments of ML algorithms, the possibilities of transferring knowledge from existing models in other domains (transfer learning) to cope with small amounts of available data [128] and growing possibilities of creating more complex models will certainly help moving closer to the dream of on-the-fly, real-time adaptive radiation therapy.

\section{Conclusion}

The treatment of moving targets with scanned particle beams is in the exciting phase of clinical implementation. To establish standards and to ensure a safe and efficient implementation, an active exchange of physicians, physicists and technicians working on this topic is required. Furthermore, a close collaboration with industry is mandatory. The annual 4D workshop provides a platform for detailed discussion on 4D imaging, motion monitoring, motion modeling, 4D planning approaches and 4D dosimetry. Furthermore, it is dedicated to introduce new approaches and techniques to further optimize the treatment of moving targets.
We are proud to organize the 10th edition of the 4D workshop in Sapporo, Japan at the end of 2018. This special venue has been made possible due to financial support from the Hokkaido University Global Station for Quantum Medical Science and Engineering (GI-CoRE, a project between Hokkaido University and Stanford University) and the University Medical Center Groningen (UMCG). If you are interested in receiving further information, please send an email to 4Dworkshop. aknopf@gmail.com.

\section{Acknowledgement}

The financial support by the Federal Ministry for Digital and Economic Affairs and the National Foundation for Research, Technology and Development is gratefully acknowledged.

\section{References}

[1] Actis O, Meer D, König S, Weber DC, Mayor A. A comprehensive and efficient daily quality assurance for PBS proton therapy. Phys Med Biol 2017;62:1661-75.

[2] Agostinelli S, Allison J, Amako K, Apostolakis J, Araujo H, Arce P, et al. GEANT4 A simulation toolkit. Nucl Instrum Methods Phys Res 2003;506:250-303.

[3] Aznar MC, Persson GF, Kofoed IM, Nygaard DE, Korreman SS. Irregular breathing during 4DCT scanning of lung cancer patients: is the midventilation approach robust? Phys Med 2014:30:69-75.

[4] Behzadi C, Groth M, Henes FO, Schwarz D, Deibele A, Begemann PGC, et al. Intraindividual comparison of image quality using retrospective and prospective respiratory gating for the acquisition of thin sliced four dimensional multidetector CT of the thorax in a porcine model. Exp Lung Res 2015;41:489-98.

[5] Bernatowicz K, Geets X, Barragan A, Janssens G, Souris K, Sterpin E. Feasibility of online IMPT adaptation using fast, automatic and robust dose restoration. Phys Med Biol 2018;63:85018.

[6] Bernatowicz K, Lomax AJ, Knopf A. Comparative study of layered and volumetric rescanning for different scanning speeds of proton beam in liver patients. Phys Med Biol 2013;58:7905-20.

[7] Bernatowicz K, Peroni M, Perrin R, Weber DC, Lomax A. Four-dimensional dose reconstruction for scanned proton therapy using liver 4DCT-MRI. Int J Radiat Oncol Biol Phys 2016;95:216-23.

[8] Bert C, Graeff C, Riboldi M, Nill S, Baroni G, Knopf A. Advances in 4D treatment planning for scanned particle beam therapy - report of dedicated workshops. Technol Cancer Res Treat 2014;13:485-95.

[9] Bert C, Grözinger SO, Rietzel E. Quantification of interplay effects of scanned particle beams and moving targets. Phys Med Biol 2008:53:2253-65.

[10] Bolwin K, Czekalla B, Frohwein LJ, Büther F, Schäfers KP. Anthropomorphic thorax phantom for cardio-respiratory motion simulation in tomographic imaging Anthropomorphic thorax phantom for cardio-respiratory motion simulation in tomographic imaging. Phys Med Biol 2018;63:035009.

[11] Boye D, Lomax T, Knopf A. Mapping motion from 4D-MRI to 3D-CT for use in 4D dose calculations: a technical feasibility study. Med Phys 2013;40:061702.

[12] Cervino L, Soultan D, Cornell M, Yock A, Pettersson N, Song WY, et al. A novel 3Dprinted phantom insert for 4D PET/CT imaging and simultaneous integrated boost radiotherapy. Med Phys 2017;44:5467-74.

[13] Chang JY, Li H, Zhu XR, Liao Z, Zhao L, Liu A, et al. Clinical implementation of intensity modulated proton therapy for thoracic malignancies. Int J Radiat Oncol Biol Phys 2014;90:809-18.

[14] Chang JY, Zhang X, Knopf A, Li H, Mori S, Dong L, et al. Consensus guidelines for implementing pencil-beam scanning proton therapy for thoracic malignancies on behalf of the PTCOG thoracic and lymphoma subcommittee. Int J Radiat Oncol Biol Phys 2017;99:41-50.

[15] Chin E, Otto K. Investigation of a novel algorithm for true 4D-VMAT planning with comparison to tracked, gated and static delivery. Med Phys 2011;38:2698-707.

[16] Cho B, Poulsen PR, Sawant A, Ruan D, Keall PJ. Real-time target position estimation using stereoscopic kilovoltage/megavoltage imaging and external respiratory monitoring for dynamic multileaf collimator tracking. Int J Radiat Oncol Biol Phys 2011;79:269-78.

[17] Dawson LA, Brock KK, Kazanjian S, Fitch D, McGinn CJ, Lawrence TS, et al. The reproducibility of organ position using active breathing control (ABC) during liver radiotherapy. Int J Radiat Oncol Biol Phys 2001;51:1410-21.

[18] De Kruijf WJM, Verstraete J, Neustadter D, Corn BW, Hol S, Venselaar JLM, et al. Patient positioning based on a radioactive tracer implanted in patients with localized prostate cancer: a performance and safety evaluation. Int J Radiat Oncol Biol Phys 2013;85:555-60.

[19] Diwanji TP, Mohindra P, Vyfhuis M, Snider III JW, Kalavagunta C, Mossahebi S, et al. Advances in radiotherapy techniques and delivery for non-small cell lung cancer: benefits of intensity-modulated radiation therapy, proton therapy, and stereotactic body radiation therapy. Transl Lung Cancer Res 2017;6:131-47.

[20] Durante M, Paganetti H. Nuclear physics in particle therapy: a review. Rep Prog Phys 2016;79:96702.

[21] Eccles CL, Patel R, Simeonov AK, Lockwood G, Haider M, Dawson LA. Comparison of liver tumor motion with and without abdominal compression using cine-magnetic resonance imaging. Int J Radiat Oncol Biol Phys 2011;79:602-8. 
[22] Ehrbar S, Jöhl A, Tartas A, Stark LS, Riesterer O, Klöck S, et al. ITV, mid-ventilation, gating or couch tracking - A comparison of respiratory motion-management techniques based on 4D dose calculations. Radiother Oncol 2017;124:80-8.

[23] Eichhorn AV. In-Vivo Feasibility Study and Developments for Cardiac Arrhythmia Ablation using Scanned Carbon Ions. Technische Universität Darmstadt; 2017.

[24] Eley JG, Newhauser WD, Richter D, Lüchtenborg R, Saito N, Bert C. Robustness of target dose coverage to motion uncertainties for scanned carbon ion beam tracking therapy of moving tumors. Phys Med Biol 2015;60:1717-40.

[25] Engwall E, Glimelius L, Hynning E. Effectiveness of different rescanning techniques for scanned proton radiotherapy in lung cancer patients. Phys Med Biol 2018;63:095006.

[26] Falk M, af Rosenschöld PM, Keall P, Cattell H, Cho BC, Poulsen P, Povzner S, Sawant A, Zimmerman J, Korreman S. Real-time dynamic MLC tracking for inversely optimized arc radiotherapy. Radiother Oncol 2010;94:218-23.

[27] Fassi A, Ivaldi GB, Meaglia I, Porcu P, de Fatis PT, Liotta M, et al. Reproducibility of the external surface position in left-breast DIBH radiotherapy with spirometerbased monitoring. J Appl Clin Med Phys 2014;15:130-40.

[28] Fassi A, Schaerer J, Fernandes M, Riboldi M, Sarrut D, Baroni G. Tumor tracking method based on a deformable 4D CT breathing motion model driven by an external surface surrogate. Int J Radiat Oncol Biol Phys 2014:88:182-8.

[29] Ferrante E, Dokania PK, Marini R, Paragios N. Deformable Registration Through Learning of Context-Specific Metric Aggregation. In: 8th International Workshop on Machine Learning in Medical Imaging. 2017. p. 256-65.

[30] Fledelius W, Keall PJ, Cho B, Yang X, Morf D, Scheib S, et al. Tracking latency in image-based dynamic MLC tracking with direct image access. Acta Oncol 2011;50:952-9.

[31] Fracchiolla F, Lorentini S, Widesott L, Schwarz M. Characterization and validation of a Monte Carlo code for independent dose calculation in proton therapy treatments with pencil beam scanning. Phys Med Biol 2015;60:8601-19.

[32] Fuchs H, Moser P, Gröschl M, Georg D. Magnetic field effects on particle beams and their implications for dose calculation in MR-guided particle therapy. Med Phys 2017;44:1149-56.

[33] Furukawa T, Hara Y, Mizushima K, Saotome N, Tansho R, Saraya Y, et al. Development of NIRS pencil beam scanning system for carbon ion radiotherapy. Nucl Instrum Methods Phys Res 2017;406:361-7.

[34] Furukawa T, Inaniwa T, Sato S, Shirai T, Takei Y, Takeshita E, et al. Performance of the NIRS fast scanning system for heavy-ion radiotherapy. Med Phys 2010;37:5672-82.

[35] GE Healthcare. Smart Deviceless 4D. https://www.gehealthcare.com/en/ products/computed-tomography/radiation-therapy-planning/smart-deviceless-4d. 2018.

[36] Ghesu FC, Georgescu B, Mansi T, Neumann D, Hornegger J, Comaniciu D. An Artificial Agent for Anatomical Landmark Detection in Medical Images. In: Medical Image Computing and Computer-Assisted Intervention. 2016. p. 229-37.

[37] Gorjiara T, Kuncic Z, Doran S, Adamovics J, Baldock C. Water and tissue equivalence of a new PRESAGE formulation for 3D proton beam dosimetry using Monte Carlo method. Med Phys 2012;39:7071-9.

[38] Graeff C. Motion mitigation in scanned ion beam therapy through 4D-optimization. Physica Med 2014;30:570-7.

[39] Graeff C, Lüchtenborg R, Eley JG, Durante M, Bert C. A 4D-optimization concept for scanned ion beam therapy. Radiother Oncol 2013;109:419-24.

[40] Grimwood A, McNair HA, O'Shea TP, Gilroy S, Thomas K, Bamber JC, Tree AC, Harris EJ. In vivo validation of Elekta's clarity autoscan for ultrasound-based in trafraction motion estimation of the prostate during radiation therapy. Int J Radiat Oncol Biol Phys 2018. In press.

[41] Haas O, Mills J, Land I, Mulholl P, Menary P, Crichton R, et al. IGRT/ART phantom with programmable independent rib cage and tumor motion. Med Phys 2014;41:022106

[42] Hara Y, Furukawa T, Mizushima K, Inaniwa T, Saotome N, Tansho R, et al. Commissioning of full energy scanning irradiation with carbon-ion beams ranging from 55.6 to $430 \mathrm{MeV} / \mathrm{u}$ at the NIRS-HIMAC. Nucl Instrum Methods Phys Res 2017:406:343-6.

[43] Hartman J, Kontaxis C, Bol GH, Frank SJ, Lagendijk JJW, Van Vulpen M, et al. Dosimetric feasibility of intensity modulated proton therapy in a transverse magnetic field of 1.5 T. Phys Med Biol 2015;60:5955-69.

[44] Heinzerling JH, Anderson JF, Papiez L, Boike T, Chien S, Zhang G, et al. Fourdimensional computed tomography scan analysis of tumor and organ motion at varying levels of abdominal compression during stereotactic treatment of lung and liver. Int J Radiat Oncol Biol Phys 2008;70:1571-8.

[45] Ibragimov B, Xing L. Segmentation of organs-at-risks in head and neck CT images using convolutional neural networks. 44: 2017; p. 547-57.

[46] Inaniwa $T$. Development of treatment planning software for carbon-ion scanning at HIMAC. In: Proceedings of HIAT0. 2009.

[47] Iwata Y. Mutliple-energy operation with quasi-dc extension of flattops at HIMAC. In: Proceedings of IPAC'10. 2010.

[48] Jeraj R, Keall P. Monte Carlo-based inverse treatment planning. Phys Med Biol 1999;44:1885-96.

[49] Jeraj R, Keall P. The effect of statistical uncertainty on inverse treatment planning based on Monte Carlo dose calculation. Phys Med Biol 2000;45:3601-13.

[50] Jia X, Ziegenhein P, Jiang SB. GPU-based high-performance computing for radiation therapy. Phys Med Biol 2014;59:R151-82.

[51] Jochems A, Deist TM, El Naqa I, Kessler M, Mayo C, Reeves J, et al. Developing and validating a survival prediction model for nsclc patients through distributed learning across 3 countries. Int J Radiat Oncol Biol Phys 2017;99:344-52.

[52] Kang M, Huang S, Solberg TD, Mayer R, Thomas A, Teo BKK, et al. A study of the beam-specific interplay effect in proton pencil beam scanning delivery in lung cancer. Acta Oncol 2017;56:531-40

[53] Keall P, Siebers J, Joshi S, Mohan R. Monte Carlo as a four-dimensional radiotherapy treatment-planning tool to account for respiratory motion. Phys Med Biol 2004;49:3639-48.

[54] Klimpki GM. Continuous irradiations in proton therapy: balancing risks and benefits. ETH Zürich; 2018.

[55] Knopf A, Bert C, Heath E, Nill S, Kraus K, Richter D, et al. Special report: workshop on 4D-treatment planning in actively scanned particle therapy-Recommendations, technical challenges, and future research directions. Med Phys 2010;37:4608-14.

[56] Knopf A, Nill S, Yohannes I, Graeff C, Dowdell S, Kurz C, Sonke J-J, Biegun AK, Lang S, McClelland J, Champion B, Fast M, Wölfelschneider J, Gianoli C, Rucinski A, Baroni G, Richter C, van de Water S, Grassberger C, Weber D, Poulsen P,

Shimizu S, Bert C. Challenges of radiotherapy: report on the 4D treatment planning workshop 2013. Phys Med 2014;30:809-15.

[57] Knopf AC, Hong TS, Lomax A. Scanned proton radiotherapy for mobile targets The effectiveness of re-scanning in the context of different treatment planning approaches and for different motion characteristics. Phys Med Biol 2011;56:7257-71.

[58] Knopf AC, Stützer K, Richter C, Rucinski A, da Silva J, Phillips J, et al. Required transition from research to clinical application: report on the 4D treatment planning workshops 2014 and 2015. Phys Med 2016;32:874-82.

[59] Kostiukhina N, Georg D, Rollet S, Kuess P, Sipaj A, Andrzejewski P, et al. Advanced radiation DOSimetry phantom (ARDOS): a versatile breathing phantom for 4D radiation therapy and medical imaging. Phys Med Biol 2017;62:8136-53.

[60] Kubiak T. Particle therapy of moving targets-the strategies for tumour motion monitoring and moving targets irradiation. Br J Radiol 2016;89:20150275.

[61] Lens E, Gurney-Champion OJ, Tekelenburg DR, van Kesteren Z, Parkes MJ, van Tienhoven G, et al. Abdominal organ motion during inhalation and exhalation breath-holds: pancreatic motion at different lung volumes compared. Radiother Oncol 2016;121:268-75.

[62] Li X, Wang X, Li Y, Zhang X. A 4D IMRT planning method using deformable image registration to improve normal tissue sparing with contemporary delivery tech niques. Radiat Oncol 2011;6:83.

[63] Lin L, Huang S, Kang M, Hiltunen P, Vanderstraeten R, Lindberg J, et al. A benchmarking method to evaluate the accuracy of a commercial proton monte carlo pencil beam scanning treatment planning system. J Appl Clin Med Phys 2017:18:44-9.

[64] Lin L, Kang M, Solberg TD, Mertens T, Baumer C, Ainsley CG, et al. Use of a nove two-dimensional ionization chamber array for pencil beam scanning proton therapy beam quality assurance. J Appl Clin Med Phys 2015;16:270-6.

[65] Liu W, Schild SE, Chang JY, Liao Z, Chang Y-H, Wen Z, et al. Exploratory study of 4D versus $3 \mathrm{D}$ robust optimization in intensity modulated proton therapy for lung cancer. Int J Radiat Oncol Biol Phys 2016;95:523-33.

[66] Lopatiuk-Tirpak O, Su Z, Li Z, Zeidan OA, Meeks SL, Maryanski MJ. Direct response to proton beam linear energy transfer (LET) in a novel polymer gel dosimeter formulation. Technol Cancer Res Treat 2012;11:441-5.

[67] Ma Y, Chang D, Keall P, Xie Y, Park JY, Suh TS, et al. Inverse planning for fourdimensional (4D) volumetric modulated arc therapy. Med Phys 2010;37:5627-33.

[68] Ma Y, Lee L, Keshet O, Keall P, Xing L. Four-dimensional inverse treatment planning with inclusion of implanted fiducials in IMRT segmented fields. Med Phys 2009;36:2215-21.

[69] Markel D, Levesque I, Larkin J, Léger P, El Naqa I. A 4D biomechanical lung phantom for joint segmentation/registration evaluation. Phys Med Bio 2016;61:7012-30.

[70] Mayer R, Liacouras P, Thomas A, Kang M, Lin L, Simone CB. 3D printer generated thorax phantom with mobile tumor for radiation dosimetry. Rev Sci Instrum 2015;86:074301.

[71] McClelland JR, Hawkes DJ, Schaeffter T, King AP. Respiratory motion models: a review. Med Image Anal 2013;17:19-42.

[72] McClelland JR, Modat M, Arridge S, Grimes H, D'Souza D, Thomas D, et al. A generalized framework unifying image registration and respiratory motion models and incorporating image reconstruction, for partial image data or full images. Phys Med Biol 2017;62:4273-92.

[73] Meyer P, Noblet V, Mazzara C, Lallement A. Survey on deep learning for radiotherapy. Comput Biol Med 2018;98:126-46.

[74] Miao S, Wang ZJ, Zheng Y, Liao R. Real-time 2D/3D registration via CNN regression. In: 2016 IEEE 13th International Symposium on Biomedical Imaging. 2016. p. $1430-34$

75] Mitchell TM. Machine learning. New York: McGraw-Hill; 1997.

[76] Mizushima K, Furukawa T, Iwata Y, Hara Y, Saotome N, Saraya Y, et al Performance of the HIMAC beam control system using multiple-energy synchrotron operation. Nucl Instrum Methods Phys Res 2017;406:347-51.

[77] Mori S, Takei Y, Shirai T, Hara Y, Furukawa T, Inaniwa T, et al. Scanned carbonion beam therapy throughput over the first 7 years at National Institute of Radiological Sciences. Phys Med 2018;52:18-26.

[78] Mori S, Zenklusen S, Inaniwa T, Furukawa T, Imada H, Shirai T, et al. Conformity and robustness of gated rescanned carbon ion pencil beam scanning of liver tumors at NIRS. Radiother Oncol 2014;111:431-6.

[79] Mostafaei F, Tai A, Gore E, Johnstone C, Haase W, Ehlers C, et al. Feasibility of real-time lung tumor motion monitoring using intrafractional ultrasound and $\mathrm{kV}$ cone beam projection images. Med Phys 2018. In press.

[80] Moteabbed M, Schuemann J, Paganetti H. Dosimetric feasibility of real-time MRI guided proton therapy. Med Phys 2014;41:1-11.

[81] Munoz L, Ziebell A, Morton J, Bhat M. An open source solution for an in-house built dynamic platform for the validation of stereotactic ablative body radiotherapy for VMAT and IMRT. Australas Phys Eng Sci Med 2016;39:957-64. 
[82] Ng JA, Booth JT, Poulsen PR, Fledelius W, Worm ES, Eade T, et al. Kilovoltage intrafraction monitoring for prostate intensity modulated arc therapy: first clinical results. Int J Radiat Oncol Biol Phys 2012;84:e655-61.

[83] Nguyen D, Long T, Jia X, Lu W, Gu X, Iqbal Z, Jiang S. Dose Prediction with U-net: A Feasibility Study for Predicting Dose Distributions from Contours using Deep Learning on Prostate IMRT Patients. 2017.

[84] Noda K. Recent progress on HIMAC for carbon therapy. In: Proceedings of PAC09. 2009.

[85] Oborn BM, Dowdell S, Metcalfe PE, Crozier S, Mohan R, Keall PJ. Proton beam deflection in MRI fields: implications for MRI-guided proton therapy. Med Phys 2015;42:2113-24.

[86] Oborn BM, Dowdell S, Metcalfe PE, Crozier S, Mohan R, Keall PJ, et al. Future of medical physics: real-time MRI guided proton therapy. Med Phys 2017;44:e77-90.

[87] Papiez L, Rangaraj D, Keall P. Real-time DMLC IMRT delivery for mobile and deforming targets. Med Phys 2005;32:3037-48.

[88] Parodi K, Mairani A, Brons S, Hasch BG, Sommerer F, Naumann J, et al. Monte Carlo simulations to support start-up and treatment planning of scanned proton and carbon ion therapy at a synchrotron-based facility. Phys Med Biol 2012;57:3759-84.

[89] Perrin RL, Zakova M, Peroni M, Bernatowicz K, Bikis C, Knopf AK, et al. An anthropomorphic breathing phantom of the thorax for testing new motion mitigation techniques for pencil beam scanning proton therapy. Phys Med Biol 2017;62:2486-504.

[90] Phillips MH, Pedroni E, Blattmann H, Boehringer T, Coray A, Scheib S. Effects of respiratory motion on dose uniformity with a charged particle scanning method. Phys Med Biol 1992;37:223.

[91] Piersanti L, Bellini F, Bini F, Collamati F, De Lucia E, Durante M, et al. Measurement of charged particle yields from PMMA irradiated by a $220 \mathrm{MeV} / \mathrm{u}$ 12C beam. Phys Med Biol 2014;59:1857-72.

[92] Pollock S, Kipritidis J, Lee D, Bernatowicz K, Keall P. The impact of breathing guidance and prospective gating during thoracic 4DCT imaging: an XCAT study utilizing lung cancer patient motion. Phys Med Biol 2016;61:6485-501.

[93] Poulsen PR, Eley J, Langner U, Simone CB, Langen K. Efficient interplay effect mitigation for proton pencil beam scanning by spot-adapted layered repainting evenly spread out over the full breathing cycle. Int J Radiat Oncol Biol Phys 2018;100:226-34.

[94] Raaymakers BW, Raaijmakers AJE, Lagendijk JJW. Feasibility of MRI guided proton therapy: magnetic field dose effects Related content Feasibility of MRI guided proton therapy: magnetic field dose effects. Phys Med Biol 2008;53:5615-22.

[95] Ravkilde T, Keall PJ, Højbjerre K, Fledelius W, Worm E, Poulsen PR. Geometric accuracy of dynamic MLC tracking with an implantable wired electromagnetic transponder. Acta Oncol 2011;50:944-51.

[96] Rietzel E, Chen G, Choi N, Willet C. Four-dimensional image-based treatment planning: target volume segmentation and dose calculation in the presence of respiratory motion. Int J Radiat Oncol Biol Phys 2005;61:1535-50.

[97] Rit S, Wolthaus J, van Herk M, Sonke J. On-the-fly motion-compensated conebeam ct using an a priori model of the respiratory motion. Med Phys 2009;36:2283-96.

[98] Rosu M, Balter JM, Chetty IJ, Kessler ML, McShan DL, Balter P, et al. How extensive of a 4D dataset is needed to estimate cumulative dose distribution plan evaluation metrics in conformal lung therapy? Med Phys 2007;34:233-45.

[99] Saini J, Maes D, Egan A, Bowen SR, St James S, Janson M, et al. Dosimetric evaluation of a commercial proton spot scanning Monte-Carlo dose algorithm: comparisons against measurements and simulations. Phys Med Biol 2017;62:7659-81.

[100] Sato S, Furukawa T, Noda K. Development of intensity control system with rfknockout extraction at the HIMAC synchrotron. In: Proceedings of EPAC. 2006

[101] Sawant A, Smith RL, Venkat RB, Santanam L, Cho B, Poulsen P, et al. Toward submillimeter accuracy in the management of intrafraction motion: the integration of real-time internal position monitoring and multileaf collimator target tracking. Int J Radiat Oncol Biol Phys 2009;74:575-82.

[102] Sawant A, Venkat R, Srivastava V, Carlson D, Povzner S, Cattell H, et al. Management of three-dimensional intrafraction motion through real-time DMLC tracking. Med Phys 2008;35:2050-61.

[103] Schätti A, Meer D, Lomax AJ. First experimental results of motion mitigation by continuous line scanning of protons. Phys Med Biol 2014;59:5707-23.

[104] Schätti A, Zakova M, Meer D, Lomax AJ. Experimental verification of motion mitigation of discrete proton spot scanning by re-scanning. Phys Med Biol 2013;58:8555-72.

[105] Schiavi A, Senzacqua M, Pioli S, Mairani A, Magro G, Molinelli S, et al. Fred: a GPU-accelerated fast-Monte Carlo code for rapid treatment plan recalculation in ion beam therapy. Phys Med Biol 2017;62:7482-504.

[106] Schlaefer A, Fisseler J, Dieterich S, Shiomi H, Cleary K, Schweikard A. Feasibility of four-dimensional conformal planning for robotic radiosurgery. Med Phys 2005;32:3786-92.

[107] Schoemers C, Feldmeier E, Naumann J, Panse R, Peters A, Haberer T. The intensity feedback system at Heidelberg Ion-Beam Therapy Centre. Nucl Instrum Methods Phys Res 2015;795:92-9.

[108] Schweikard A, Shiomi H, Adler J. Respiration tracking in radiosurgery. Med Phys 2004;31:2738-41.

[109] Souris K, Lee JA, Sterpin E. Fast multipurpose Monte Carlo simulation for proton therapy using multi-and many- core CPU architectures. Med Phys 2016;43:1700-2366.

[110] Stieler F, Wenz F, Lohr F. Laser tracking vs CBCT for patient positioning. Radiother Oncol 2012;103:S542.

[111] Taylor PA, Kry SF, Followill DS. Pencil beam algorithms are unsuitable for proton dose calculations in lung. Int J Radiat Oncol Biol Phys 2017;99:750-6.

[112] Testa M, Verburg JM, Rose M, Min CH, Tang S, Bentefour EH, et al. Proton radiography and proton computed tomography based on time-resolved dose measurements. Phys Med Biol 2013;58:8215-33.

[113] Tewatia D, Zhang T, Tome W, Paliwal B, Metha M. Clinical implementation of target tracking by breathing synchronized delivery. Med Phys 2006;33:4330-6.

[114] Thomas D, Lamb J, White B, Jani S, Gaudio S, Lee P, et al. A novel fast helical 4DCT acquisition technique to generate low-noise sorting artifact-free images at userselected breathing phases. Int J Radiat Oncol Biol Phys 2014;89:191-8.

[115] Von Siebenthal M, Sźkely G, Lomax AJ, Cattin PC. Systematic errors in respiratory gating due to intrafraction deformations of the liver. Med Phys 2007;34:3620-9.

[116] Welsh J, Amini A, Ciura K, Nguyen N, Palmer M, Allen PK, et al. Evaluating proton stereotactic body radiotherapy to reduce chest wall dose in the treatment of lung cancer. Med Dosim 2013;38:442-7.

[117] Winterhalter C, Fura E, Tian Y, Aitkenhead A, Bolsi A, Dieterle M, et al. Validating a Monte Carlo approach to absolute dose quality assurance for proton pencil beam scanning. Phys Med Biol 2018;63:175001.

[118] Wolf R, Bortfeld T. An analytical solution to proton Bragg peak deflection in a magnetic field. Phys Med Biol 2012;57:N329-37.

[119] Xiang L, Wang Q, Nie D, Zhang L, Jin X, Qiao Y, et al. Deep embedding convolutional neural network for synthesizing CT image from T1-Weighted MR image. Med Image Anal 2018;47:31-44.

[120] Yohannes I, Hild S, Vasiliniuc S, Langner O, Graeff C, Bert C. Technical Note: radiation properties of tissue- and water-equivalent materials formulated using the stoichiometric analysis method in charged particle therapy. Med Phys 2016;43:308-13

[121] Yu C-NJ, Joachims T. Learning structural SVMs with latent variables. In: Proceedings of the 26th Annual International Conference on Machine Learning ICML '09. 2009. p. 1-8.

[122] Yu ZH, Lin SH, Balter P, Zhang L, Dong L. A comparison of tumor motion characteristics between early stage and locally advanced stage lung cancers. Radiother Oncol 2012;104:33-8.

[123] Zeng R, Fessler JA, Balter JM, Balter PA. Iterative sorting for four-dimensional CT images based on internal anatomy motion. Med Phys 2008;35:917-26.

[124] Zenklusen SM, Pedroni E, Meer D. A study on repainting strategies for treating moderately moving targets with proton pencil beam scanning at the new gantry 2 at PSI. Phys Med Biol 2010;55:5103-21.

[125] Zhang Y, Boye D, Tanner C, Lomax AJ, Knopf A. Respiratory liver motion estimation and its effect on scanned proton beam therapy. Phys Med Biol 2012;57:1779-95

[126] Zhang Y, Huth I, Weber D, Lomax A. A statistical comparison of motion mitigation performances and robustness of various pencil beam scanned proton systems for liver tumour treatments. Radiother Oncol 2018. in press.

[127] Zhao L, Newton J, Oldham M, Das IJ, Cheng C-W, Adamovics J. Feasibility of using PRESAGE ${ }^{\circledR}$ for relative 3D dosimetry of small proton fields. Phys Med Biol 2012:57:N431-43.

[128] Zhen X, Chen J, Zhong Z, Hrycushko B, Zhou L, Jiang S, et al. Deep convolutional neural network with transfer learning for rectum toxicity prediction in cervica cancer radiotherapy: a feasibility study. Phys Med Biol 2017;62:8246-63.

[129] Zhu XR, Li Y, Mackin D, Li H, Poenisch F, Lee AK, et al. Towards effective and efficient patient-specific quality assurance for spot scanning proton therapy. Cancers 2015;7:631-47.

[130] Zhu XR, Poenisch F, Song X, Johnson JL, Ciangaru G, Taylor MB, et al. Patientspecific quality assurance for prostate cancer patients receiving spot scannin proton therapy using single-field uniform dose. Int J Radiat Oncol Biol Phys 2011;81:552-9.

[131] Zhu Z, Liu W, Gillin M, Gomez DR, Komaki R, Cox JD, et al. Assessing the robustness of passive scattering proton therapy with regard to local recurrence in stage III non-small cell lung cancer: a secondary analysis of a phase II trial. Radiat Oncol 2014;9:108. 